\title{
LIPOSOME AS A DRUG DELIVERY CARRIER-A REVIEW
}

\author{
Available online at www.ijdra.com \\ REVIEW ARTICLE \\ * Kumari Rekha, Varma Kuldeep, Srivastava Surabhi, Jyoti Rai Prachya, Maurya Sheo Datta \\ Department of Pharmacy, IEC Group of Institutions, Knowledge Park.-I, Greater Noida, INDIA- \\ 201308 \\ *Corresponding Author's E-mail: Rekha_baghel08@yahoo.co.in
}

\section{ABSTRACT:}

The evolution of the science and technology of liposomes as a drug carrier has passed through a number of distinct phases. Because they exhibit peculiar properties due to their structure, chemical composition amphiphilic nature, physico-chemical characters and colloidal size, which are used in various applications. These properties point to several applications as the solubilizer for insoluble drugs, dispersants, and sustained release system, delivery system for the encapsulated substance, stabilizer, protective agents, and micro reactive being the most obvious ones. Yet interest in liposomes, especially among academic workers, spread rapidly we attribute this to the remarkable structural versatility of the system, which enables the design of countless liposomes versions to satisfy particular needs in terms of both technology and optimal function in vivo.

Key words: Liposomes, Liposomal vesicles, MLV, SUV, OLV, Phospholipid.

\section{INTRODUCTION $^{1-3}$}

Liposomal vesicles were prepared in the early years of their history from various lipid classes identical to those present in most biological membranes. Liposomes were discovered in the mid of 1960' s and originally studied as cell membrane model Paul Ehrlich coined the term "magic bullet" in $20^{\text {th }}$ century where carrier system's was proposed to simply carry the drug to its of action and releasing its selectively while non target sites should absolutely be exempted from drug effect. The exploration and progressive advent of liposomal drug delivery system has rekindled interest in magic bullet approach, for surely man's ingenuity can find means for directing these drugs filled packed or

lipid bilayer vesicles to specific cell or anatomical sites within the body. Liposomes were described as a model of cellular membranes and quickly were applied to the delivery of substances to cells. Liposomes are microscopic spherical vesicles that form when phospholipids are hydrated. When mixed in water under low shear conditions, the phospholipids arrange themselves in sheets, the molecules aligning side by side in like orientation, "heads" up and "tails" down. These sheets then join tails-to-tails to form a bilayer membrane, which encloses some of the water in a phospholipids sphere

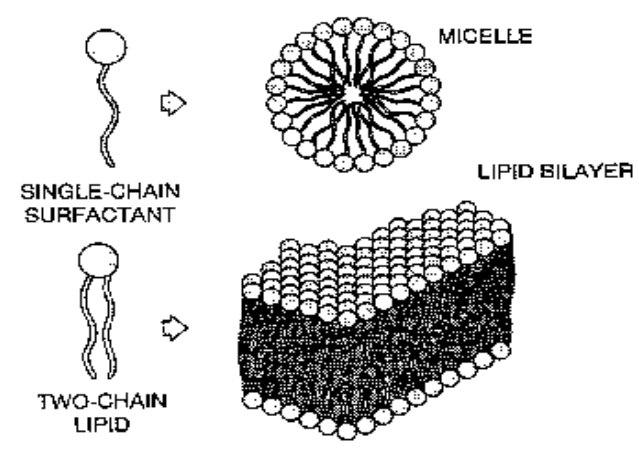

\section{Mechanism of liposomal formation ${ }^{4}$}

Liposomes are formed open hydration of lipid molecules normally lipids are hydrated from a dry state (thin or thick lipid film, spray dried powder), and stacks of crystalline bilayers become fluid and swell myelin-long, thin cylinders grow and upon agitation detach self close in to large, multilameller liposomes because this eliminates unfavorable interactions at the edges. Once the large particles are formed they can be either broken by mechanical 
treatment in to smaller bilayered fragments, which close into smaller liposomes.

\section{Classification of liposomes ${ }^{5-7}$}

Multilameller vesicles (MLV) consist of several (up to 14) lipid layers (in an onion-like arrangement) separated from one another by a layer of aqueous solution. These vesicles are over several hundred nanometers in diameter. Small unilamellar vesicles (SUV) are surrounded by a single lipid layer and are 25-50 nm (according to some authors up to $100 \mathrm{~nm}$ ) in diameter. Large unilamellar vesicles (LUV) are, in fact, a very heterogeneous group of vesicles that, like the SUVs, are surrounded by a single lipid layer. The diameter of these liposomes is very broad, from $100 \mathrm{~nm}$ up to cell size (giant vesicles) Besides the technique used for their formation the lipid composition of liposomes is also, in most cases, very important.

\section{Methods of liposomes preparation ${ }^{8,9}$}

Basic studies on liposomal vesicles resulted in numerous methods of their preparation and characterization.

\section{A. Hydration stage}

1. Mechanical methods

- Vortexing or hand shaking of phospholipid dispersions (MLV)

- 'Microfluidizer' technique (mainly SUV)

- High-shear homogenization (mainly SUV)

2. Methods based on replacement of organic solvent(s) by aqueous media

- Removal of organic solvent(s) before hydration (MLV, OLV, SUV)

- Reverse-phase evaporation (LUV, OLV, MLV)

- Use of water immiscible solvents: ether and petroleum-ether infusion (solvent vaporization) (MLV, OLV, LUV)

- Use of water miscible solvents such as ethanol injection (MLV, OLV, SUV)

3. Methods based on detergent removal

- Gel exclusion chromatography (SUV)

- 'Slow' dialysis (LUV, OLV, MLV)

- Fast dilution (LUV, OLV)

4. Methods based on size transformation and fusion
- Spontaneous fusion of SUV in the gel phase (LUV)

- Freeze-thawing (MLV)

- Freeze-drying (MLV)

B. Sizing stage

1. High pressure extrusion

2. Low pressure extrusion

3. Ultrasonic treatment

C. Removal of non-encapsulated material

1. Dialysis

2. Ultracentrifugation

3. Gel-permeation chromatography

4. Ion-exchange resins

Targeting via Liposomes ${ }^{6,10,11}$

Depending on the need, one can use SUV type or MLV type vesicles for effective entrapment and delivery of the drug to the target tissues or cells. Nevertheless, charge properties and interactions of the active compound with vesicle forming molecules will determine the effect of entrapment, i.e., the amount of the compound that can be "loaded" into a single vesicle. On the other hand, the composition of the molecules used for the formation of the vesicular structure will, at least, affect the fate of vesicles from the site of their introduction as well as the interaction with component of the body (e.g., surface charge, serum proteins, lipoproteins, opsonin system, phagocytic system and finally target cells. In the earlier studies, when therapeutically active substances were not easily available, most of the experiments were done using a marker compound. The results, however, were not the same as those obtained in experiments in which an active substance was used and the conditions were more related to the real situation (ex-vivo, in-vivo). These findings implicate the necessity for studies in which an active substance is used and the conditions of the experiments resemble, as closely as possible, those of therapeutic Liposomal (vesicular) drug application. The benefits of liposomal formulations were already demonstrated clinically and stimulate many laboratories (research and pharmaceutical) in their efforts to introduce new Liposomal vesicular drugs $\mathbf{1 2}$

Liposomes are well established as drug carriers in topical treatment of diseases, especially in dermatology. They can enhance penetration of encapsulated hydrophilic drugs into the skin to 
enable a proper therapeutic effect. Because of they are able to carry with them any enclosed substances into the dermis and to 'the individual cells ${ }^{13}$

\section{Sustained release of the incorporated drug ${ }^{14,15}$}

Liposomes are typical vehicles, which are able to transport dermatological and cosmetic active agents of different types. The active agents are encapsulated und protected against environmental influences. Liposomes spread out excellently in the horny layer of the skin and form depots of active agents. Aqueous dispersions show a clear to milky appearance according to the size of the Liposomes. Similar to the horny layer of human skin, liposomes consist of one or several bilayers of phosphatidylcholine. Liposomes without active agents ("empty liposomes") show all dermatological and cosmetic effects of phosphatidylcholine.

The size of these spheres is very small, in the order of a nanometer. As illustrated, the spheres are hollow inside and enclose some of the liquid material in which they were formed (inclusion). Because of the small size of the phospholipid molecule and microspheres, they can pass through the epidermis and act as a carrier for the enclosed substances.

\section{Release Kinetics of Liposomal Payload ${ }^{4}$}

Liposomes are most useful for being able to transfer and deliver active ingredients to the application site of formulation. The liposome wall is very similar, physiologically, to the material of cell membranes. Application of formulation over skin area causes deposition of liposomes on the skin and begins to merge with the cellular membranes. In the process, the liposomes release their payload of active materials into the cells. As a consequence, not only is delivery of the actives very specific directly into the intended cells but also the delivery takes place over a longer period of time. Liposomes exhibit better stability, penetration and efficacy at lower usage levels.

Liposomes as a delivery system can be made to release their payload under a variety of conditions.

- Slow / Fast Release of Hydrophilic Payload

- Slow / Fast Release of Hydrophobic Payload

- Bilayer Composition
1) Chain Length

2) Saturation

3) Lipid Class

- Physical Configuration of Liposome

- Solvent-Dependent Release

- $\mathrm{pH}$-Dependent Release

- Temperature-Dependent Release

Advantages of Liposomes ${ }^{16}$

The characteristics of liposomes also yield a variety of other formulation benefits.

- Controlled Delivery System

- Biodegradable, Non-Toxic

- Carry Both Water and Oil Soluble Payloads

- Can Solubilize Recalcitrant Compounds

- Prevention of Oxidation

- Protein Stabilization

- Controlled Hydration

\section{Applicability of Liposomes}

One may conclude that, at present, the term "liposomes" covers not only phospholipid based vesicles but also other vesicular structures with properties identical or similar to those of classical, natural phospholipid based Liposomes. In the early 70's the use of liposomes as a drug carrier system was proposed by Gregoriadis \& Ryman. Since this first report, liposomes were developed as an advanced drug delivery vehicle. They are generally considered non-toxic, biodegradable and non-immunogenic (Osborne D. W. et al.). Associating a drug with liposomes markedly changes its pharmacokinetics and lowers systemic toxicity; furthermore, the drug is prevented from early degradation and/or inactivation after introduction to the target organism (Gabizon A. et al.). The use of liposomes or, in general, vesicular structures for the delivery of various active compounds is recognized in relation to water solubility of the compound. When the compound is water soluble, the size and volume of the aqueous compartment of the vesicle is crucial (Daan J. A. et al.). In contrast, hydrophobic compounds will prefer incorporation into the lipid (amphiphile) layer that constructs the vesicle. In such a case, the size of the aqueous compartment is not important. 


\section{References}

\section{Therapeutic applications of liposomes}

Liposomes are used for the following range of therapeutic and pharmaceutical applications (Vyas .S P. et al.):

1. Liposomes as drug protein delivery vehicles.

-Controlled and sustained drug release in situ.

-Enhanced drug solubilization.

-Altered pharmacokinetics and biodistribution.

-Enzyme replacement therapy and lysosomal storage disorders

2. Liposomes in antimicrobial and antifungal (lung therapeutics) and antiviral (anti-HIV) therapy

- Liposomal drugs

- Liposomal biological response modifiers

3. Liposomes in tumour therapy

- Carrier of small cytotoxic molecules

- Vehicle for macromolecules as cytokines or genes

4. Liposomes in gene delivery.

- Gene and antisense therapy

- Genetic (DNA) vaccination

5. Liposome Immunology.

- Immunoadjuvant

- Immunomodulator

- Immunodiagnosis

6. Liposomes as artificial blood surrogates.

7. Liposomes as Radiopharmaceutical and Radiodiagnostic carriers

8. Liposomes in cosmetics and dermatology

9. Liposomes in enzyme immobilization and bioreactor technology.

\section{Conclusion}

Liposomes have been realized as extremely useful carrier systems, additive(s) and tools in various scientific domains. Thus, liposomes over the years have been investigated as the major drug delivery systems due to their flexibility to be tailored for varied desirable purposes. The flexibility in their behavior can be exploited for the drug delivery through any route of administration and for-any drug or material irrespective of its physicochemical properties. The uses of liposomes in the delivery of drugs and genes to tumour sites are promising and may serve as a handle for focus of future research.
1. Abraham W and Downing O. T., 1990; Biochim. Biophys. Acta 1021, 119.

2. Crommelin OJA, Schreier H. 1994: Liposomes. In: Krcuter J, cd. Colloidal Drug Delivery systems. Marcel Dekker, Inc., 73-190.

3. Barenholz Y. 2001; Liposome application: problems and prospects. Curl' Opin Colloid Interface Sci 6:66-77.

4. Lasic D D., July 1998; Novel application of liposomes, Tibtech, Vol.16.

5. Allen T. M., 1994; Trends Pharm. Sci. 15,215

6. Baidya S., Gupta B.K., 1998, High Technology site-specific approach through liposomal drug delivery system. Indian $\mathbf{J}$ pharm sci, 32 (1), 35-37.

7. Swarbrick J, Boylan JC, Cds. 1994, Encyclopedia or Pharmaceutical Technology, Vol. 9,NY: Marcel Dekker: 1-39.

8. Storm G, Crommelin DJA. Quo Vadis? 1998, Pharmaceutical Science and Technology 1:19-31.

9. Vyas .S P., Khar, R.K., 2004; Targeted and Controlled drug delivery: Novel Carrier system; first reprint edition, C.B.S. publications.

10. Bandak S, Gorcn D, Horowitz A, Tzcmach O, Gabizon A., 1999; Pharmacological studies of cisplatin encapsulated in long-circulating liposomes in mouse tumor models. Anticancer drugs 10:911-920.

11. Gabizon A, Goren O, Cohen R, Barenholz Y. 1998, Development or liposomal anthracyclines: from basics to clinical applications. J Controlled Release.

12. Lasic DD., 1993 Liposomes: From Physics to Applications. Amsterdam: Elsevier.

13. Zhang YP, Ceh B. Lasic DD. 2001:Liposomics in drug delivery. In: Polymcric Biomaterials. 2nd cd. Chapter 29. Damitziu S, ed. New York: Marcel Dekker, 783-821.

14. Zuidam NJ, Van Winden E, De Vrueh R, Crommclin DJA. 2003, In: Torehilin VP, Weissig V, eds. Liposomes: A Practical Approach. 2nd Cd. Oxford University Press.

15. Vernooij EAAM, Keltencs-van den Bosch EAAM, Underberg WJM, Crommelin DJA. Chemical hydrolysis of DOTA and DOPE in a Liposomal environment. Journal of Controlled Release 2002: 79:299-303.

16. Daan J. A. Crommelin and Gert Storm, 2003, Liposomes: From the Bench to the Bed; Journal of Liposome research Vol. 13, No. I, pp. 33-36. 